\title{
HIPASS J0731-69: Tidal Debris, or Primordial Gas Cloud?
}

\author{
Stuart D. Ryder \\ Anglo-Australian Observatory, P.O. Box 296, Epping, NSW 1710, \\ Australia \\ Bärbel S. Koribalski \\ Australia Telescope National Facility, P.O. Box 76, Epping, NSW 1710, \\ Australia
}

\begin{abstract}
We present follow-up high-resolution $\mathrm{HI}$, deep optical, and molecular observations of the intergalactic gas cloud HIPASS J0731-69, and re-examine the question of its origin in light of these new data.
\end{abstract}

\section{Background}

An intergalactic gas cloud was discovered serendipitously during the course of the H I Parkes All-Sky Survey (Barnes et al. 2001), lying at a projected distance of $180 \mathrm{kpc}$ from the asymmetric spiral galaxy NGC 2442 (Ryder et al. 2001). This object, designated HIPASS J0731-69, contains $\sim 10^{9} \mathrm{M}_{\odot}$ of $\mathrm{HI}$, or nearly $1 / 3$ as much atomic gas as NGC 2442 itself. No obvious optical counterpart could be found in the Digitised Sky Survey (DSS).

\section{Follow-up Observations and Conclusions}

HIPASS J0731-69 was observed for $4 \times 12 \mathrm{hr}$ with the Australia Telescope Compact Array $^{1}$ (ATCA) in its compact $375 \mathrm{~m}$ configuration in February 2001. At $2^{\prime}$ resolution, HIPASS J0731-69 is observed to break up into seven or so distinct, or loosely-connected clumps (Figure 1), each containing $\sim 5 \times 10^{7} \mathrm{M}_{\odot}$ of $\mathrm{HI}$. The peak $\mathrm{H}$ I surface density in any clump is $<0.3 \mathrm{M}_{\odot} \mathrm{pc}^{-2}$, well below the apparent "threshold" for star formation (Martin \& Kennicutt 2001).

The area surrounding HIPASS J0731-69 was observed for $3000 \mathrm{~s}$ in the $B$ band, using the Wide Field Imager (WFI) $8 \mathrm{~K} \times 8 \mathrm{~K}$ CCD mosaic at the prime focus of the Anglo-Australian Telescope, in January 2003. Although this data reaches much fainter levels $(B \sim 24)$ than the DSS, Fig. 1 still shows no clear optical counterparts to three of the densest H I clumps.

Each of the H I clumps identified with the ATCA was observed for up to $6 \mathrm{hr}$ with the Swedish-ESO Submillimetre Telescope (SEST) in February 2002,

\footnotetext{
${ }^{1}$ The Australia Telescope is funded by the Commonwealth of Australia for operation as a National Facility managed by CSIRO.
} 


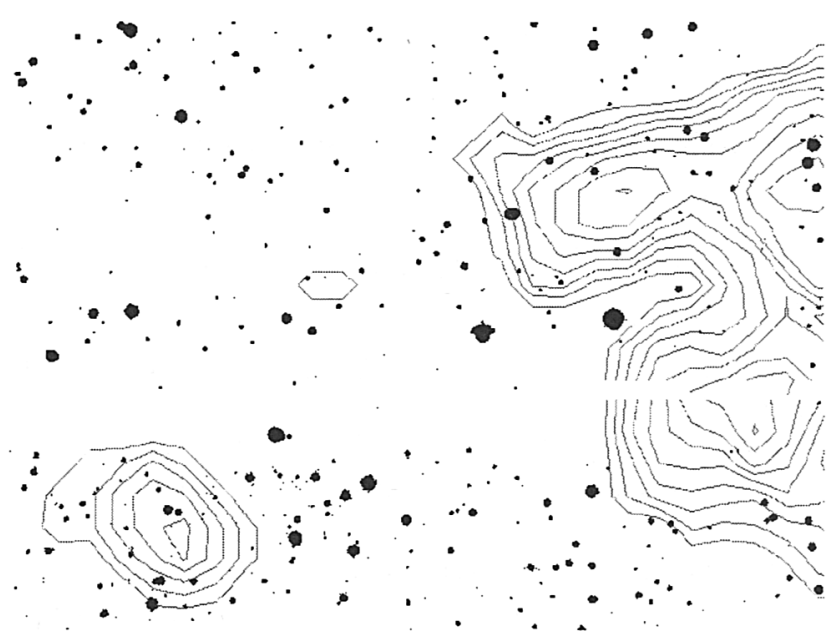

Figure 1. Hi contours from the ATCA, overlaid on $B$ data from 2 adjoining CCDs of the WFI array, for the north-eastern section of HIPASS J0731-69. The break in the contours indicates the inter-CCD gap. The field shown is $15^{\prime} \times 10^{\prime}$, and the ATCA beamsize is $2^{\prime}$.

to search for $\mathrm{CO}$ emission at $115 \mathrm{GHz}$ and $230 \mathrm{GHz}$. No CO emission was detected from any of these clumps, even though $\mathrm{CO}$ was easily detectable in NGC 2442 itself (Bajaja et al. 1995).

Following up our initial discovery, we have found that HIPASS J0731-69 in fact consists of multiple $\mathrm{H}$ I clumps, possibly within a common envelope of diffuse H I (our ATCA observations resolve out nearly $2 / 3$ of the HIPASS flux). An optical and CO search for possible Tidal Dwarf Galaxies has failed to find evidence of either past gas enrichment, or current star formation. It seems less likely therefore that the gas in HIPASS J0731-69 was once part of NGC 2442. Rather, it may well be the case that HIPASS J0731-69 represents "fossil" primordial gas, perhaps preserved over a Hubble time by the relatively low-density environment of the NGC 2442 group, which includes NGC 2434 and NGC 2397. However, more extensive observations and modeling of the group gas kinematics are required if we are to rule out either ram-pressure stripping, or a tidal origin.

\section{References}

Bajaja, E., et al. 1995, A\&AS, 114, 147

Barnes, D. G., et al. 2001, MNRAS, 322, 486

Martin, C. L., \& Kennicutt, R. C. 2001, ApJ, 555, 301

Ryder, S. D., et al. 2001, ApJ, 555, 232 